\title{
VARIAÇÕES ANGULARES DO QUADRIL, JOELHO E TORNOZELO ENTRE DOIS MÉTODOS DE AJUSTE DE ALTURA DE SELIM DA BICICLETA: ESTUDOS DE CASO
}

\author{
Rivaldo de Souza Oliveira \\ Universidade Estadual de Campinas, Campinas, São Paulo, Brasil \\ Juliana Exel Santana \\ Universidade Estadual de Campinas, Campinas, São Paulo, Brasil \\ Felipe Arruda Moura \\ Universidade Estadual de Campinas, Campinas, São Paulo, Brasil \\ Sergio Augusto Cunha \\ Universidade Estadual de Campinas, Campinas, São Paulo, Brasil
}

\begin{abstract}
Resumo
O objetivo deste trabalho consistiu em avaliar os métodos Greg Lemond e Fit Kit em variáveis cinemáticas angulares de membro inferior durante a pedalada. Foram recrutados 3 atletas de ciclismo treinados, que pedalaram por 2 minutos, em uma cadência preferida. As imagens foram obtidas por 2 câmeras filmadoras digitais em uma frequência de $300 \mathrm{~Hz}$. Marcadores foram fixados no trocânter maior, epicôndilo lateral, maléolo lateral e base do $5^{\circ}$ metatarso, e rastreados no DVideo ${ }^{\circledR}$. Foram calculados os valores máximos e mínimos dos ângulos do quadril, joelho e tornozelo em 5 ciclos. Não houve diferença entre os métodos testados. Assim, uma vez que o Fit Kit é um método oneroso, é possível a realização de ajustes utilizando um método simples, como o Greg Lemond.
\end{abstract}

Palavras-chave: Ciclismo. Membros Inferiores. Biomecânica.

\section{Introdução}

Ompetições como a prova 9 de Julho, Volta Ciclística do Estado de São Paulo, Volta de Santa Catarina e Tour de France tem atraído um grande número de pessoas a utilizarem a bicicleta para fins de competição, treinamento físico, reabilitação, como meio de transporte ou para o lazer.

Estudos recentes (ALENCAR; MATIAS, 2009; MARTINS, et al. 2007) tem mencionado um crescimento no número de usuários de bicicleta, o que atraiu pesquisadores a entenderem melhor a relação entre este equipamento e o ciclista. Martins et al. (2007) apontaram que 
a falta de informações sobre melhores formas de utilização da bicicleta tem contribuído para que os ciclistas adotem posições incorretas, em particular, com acentuada flexão ou excessiva extensão do joelho durante o movimento da pedalada. Somando estas posições a um selim ajustado mais anterior ou posteriormente, pode ocorrer uma movimentação médio-lateral excessiva do joelho e, consequentemente, uma tensão maior nos músculos posteriores da coxa. Os desajustes nos elementos da bicicleta e tipos de posturas adotadas durante a pedalada podem levar a alterações biomecânicas na pedalada. Com isso, os usuários têm maiores chances de sofrerem lesões articulares nos joelhos, tendinites lesões na região lombar e períneo (ALENCAR; MATIAS, 2009).

Adequar a altura do selim ao usuário da bicicleta é um dos mais fundamentais ajustes a serem realizados antes do início da prática. Porém, o ajuste intuitivo do selim pode causar danos a curto ou médio prazo, porém, a literatura tem proposto diferentes formas de obtenção de parâmetros para a realização destes ajustes (MESTDAGH, 1998). Isso acaba por contribuir para que pesquisadores, principalmente em áreas de estudo como a biomecânica, atentem para o desenvolvimento de metodologias capazes de quantificarem as forças aplicadas nos pedais e os ângulos articulares durante a pedalada. Entender a interação usuário-bicicleta contribui para que as análises do desempenho de atletas sejam cada vez mais específicas e ofereçam dados importantes para prescrição de sessões treinamento e de reabilitação mais adequados.

Dessa forma, alguns métodos foram desenvolvidos para melhor determinar o ajuste ideal do selim da bicicleta. Greg Lemond (BURKE; PRUITT, 2003; BURKE 1994; PEVELER et al., 2005; PEVELER; POUNDERS; BISHOP, 2007), Holmes (ALENCAR; MATIAS, 2009; HOLMES; PRUITT; WHALEN, 1994). Hamley (BURKE, 1994; PEVELER, 2008) e o método calcanhar-pedal (BURKE 1994; PEVELER et al., 2005; BAILEY; MAILLARDET; MESSENGER, 2003) são exemplos desses métodos, que não fazem uso nem de profissional especializado nem de ferramentas sofisticadas para o ajuste.

O método Greg Lemond é um dos mais estudados na literatura e faz a determinação da melhor altura de ajuste através de um cálculo simples. Um estudo verificou a influência dos métodos Greg Lemond, Hamley e calcanhar-pedal no ângulo do joelho com o pedal da bicicleta posicionado no ponto morto inferior (PEVELER et al., 2005). 
Os ângulos de 19 participantes foram aferidos através de um goniômetro. Os valores angulares obtidos para o método Greg Lemond e Hamley permaneceram acima dos valores considerados recomendados para a prevenção de lesões (entre $145^{\circ}$ e $155^{\circ}$ ) e não foram encontradas diferenças significativas quando comparados. O método calcanhar-pedal apresentou diferenças significativas quando comparado aos outros dois métodos, com uma variação dos valores de ângulo dentro da faixa adequada $\left(150^{\circ}\right)$ considerada para a prevenção de lesões. Embora o método calcanhar-pedal tenha apresentado valores angulares considerados seguros para a integridade da articulação do joelho, estudos apontam que a altura do selim pelo método Hamley maximiza o desempenho dos atletas (PEVELER et al., 2005; PEVELER et al., 2007; PEVELER, 2008).

$\mathrm{O}$ efeito dos ajustes pelo método Hamley e pelo Holmes na economia de energia foi estudado por (PEVELER, 2008). O consumo máximo de oxigênio foi comparado entre 11 ciclistas altamente treinados durante a pedalada, utilizando cada um dos ajustes. Os valores obtidos para o consumo foram menores para o mesmo valor de potência para os ciclistas quando utilizaram o método Holmes.

Diferente destes métodos citados anteriormente, o Fit Kit é uma ferramenta disponibilizada pelo mercado e utilizada por atletas, profissionais da área do esporte e fisioterapeutas para a realização de ajustes. Este método apresenta-se diferenciado por levar em consideração a aerodinâmica no conjunto ciclista-bicicleta, o conforto e o desempenho do usuário (http://bikefitkit.com). O Fit Kit é composto de ferramentas de medidas e um software. As ferramentas exigem a obtenção de valores de flexibilidade e de variáveis antropométricas dos atletas para a identificação dos parâmetros de ajuste dos componentes da bicicleta. Entretanto, o Fit Kit é um pacote de avaliação de alto custo.

Já se sabe que é importante e indispensável que qualquer tipo de praticante de ciclismo ajuste os componentes de suas bicicletas de forma a aperfeiçoar seu desempenho e minimizar riscos de lesões (BINI; HUME; CROFT, 2011). Muitos métodos têm surgido e proposto maneiras de realizar o melhor ajuste, que vão desde métodos simples que utilizam poucos recursos para os cálculos, até conjuntos sofisticados. Contudo, ainda são necessários maiores esclarecimentos científicos quanto aos resultados apresentados por estes métodos. Assim, o objetivo deste trabalho consistiu em avaliar as conseqüências 
de dois diferentes ajustes da altura de selim em variáveis cinemáticas angulares do membro inferior no movimento da pedalada.

\section{Metodologia}

\section{Participantes}

Foram recrutados e avaliados 3 atletas de ciclismo treinados e experientes em competições, do gênero masculino, com idades entre 20 e 50 anos, com estado de saúde satisfatório (fisicamente saudáveis e ativos). Os participantes possuíam as seguintes características:

Participante 1: $67 \mathrm{~kg}, 1,72 \mathrm{~m}$ e 15 anos de experiência no ciclismo. Participante 2: $67 \mathrm{~kg}, 1,66 \mathrm{~m}$ e 15 anos de experiência no ciclismo. Participante 3: $69 \mathrm{~kg}, 1,70 \mathrm{~m}$ e 8 anos de experiência no ciclismo.

Este projeto foi aprovado pelo Comitê de Ética em Pesquisa - Unicamp, parecer No 682/2011 conforme a Resolução 196/96.

Determinação da altura do selim

Os métodos de ajuste de altura de selim utilizados neste estudo foram o Greg Lemond e o conjunto Fit Kit.

A altura de ajuste utilizando o método Greg Lemond é calculada por meio da equação 1:

Altura do selim $(\mathrm{cm})=$ altura entre o chão e o púbis $x 0.883$

Para a medida da altura entre o chão e o púbis, os participantes mantiveram-se em posição ortostática e o valor foi obtido utilizandose uma fita métrica. Para a realização do ajuste, o valor encontrado para a altura do selim é medido a partir do centro de rotação do pé-devela ao topo do selim.

A altura de ajuste baseada no Fit Kit necessita da obtenção de dois parâmetros para serem inseridos no software do conjunto. O primeiro deles é o valor de ângulo formado pela linha do fêmur com a linha do tronco dos participantes. Para isto, os participantes foram posicionados deitados no chão em decúbito dorsal, com o joelho e quadril esquerdo flexionado o máximo possível. A medida deste ângulo foi feita através de um goniômetro. O segundo parâmetro de entrada no Fit Kit foi à medida de altura entre o chão e o púbis, mensurada da mesma forma que o método Greg Lemond. O ajuste para a altura encontrada é 
feito a partir do centro do eixo do pedal na posição de ponto morto inferior ao topo do selim. Todos os procedimentos do Fit Kit foram realizados por uma empresa autorizada e apenas os valores de altura do selim foram disponibilizados para a pesquisa.

Uma vez que os valores de altura do selim, para cada um dos métodos, são resultados da combinação de diferentes variáveis e ajustados a partir de pontos diferentes na bicicleta, para tornar possível a comparação entre os conjuntos de dados, foi somada a distância do comprimento do pé-de-vela $(17,25 \mathrm{~cm})$ ao do centro do eixo do pedal na posição de ponto morto inferior da bicicleta aos valores de altura de selim obtidos pelo método Greg Lemond.

\section{Protocolo de Pedalada}

Os participantes pedalaram primeiramente com o selim ajustado pelo método Greg Lemond, por dois minutos, em uma cadência preferida. Em seguida, pedalaram por mais dois minutos com o selim ajustado pelo método Fit Kit, mantendo a cadência preferida. O primeiro minuto de pedalada foi considerado um período de adaptação de cada participante ao selim, não sendo utilizado para a análise cinemática. Cada participante pedalou em uma mesma bicicleta configurada para uso em competição, que foi fixada num rolo estacionário (Ergo-Bike).

Obtenção dos dados Cinemáticos da Pedalada

As imagens foram obtidas através de duas câmeras filmadoras digitais $\left(\right.$ Casio ${ }^{\circledR}$, modelo EX-F1), em uma frequência de $300 \mathrm{~Hz}$, posicionadas no plano sagital do hemicorpo esquerdo dos participantes. As câmeras permaneceram estacionárias durante todo o experimento. A sincronização das imagens foi feita a partir de um evento visual comum identificado no par de câmeras.

De toda imagem capturada, as informações de interesse foram as relacionadas ao membro inferior esquerdo de cada participante. Para representá-lo, foram fixados marcadores retro-refletivos com $15 \mathrm{~mm}$ de diâmetro nos seguintes acidentes anatômicos: trocânter maior, epicôndilo lateral, maléolo lateral e base do $5^{\circ}$ metatarso. Para a obtenção da posição desses marcadores no espaço em função do tempo de pedalada, as imagens foram processadas e os pontos de interesse 
rastreados automaticamente no software DVideo ${ }^{\circledR}$ (FIGUEROA; LEITE; BARROS, 2003). A calibração das câmeras foi feita através da filmagem de fios de prumo com marcadores com distâncias entre si conhecidas, contemplando o volume de espaço em que foram posicionados os participantes e suas respectivas bicicletas.

A reconstrução tridimensional das coordenadas obtidas para cada marcador foi feita pelo método DLT (Direct Linear Transformation) proposto por (ABDEL-AZIZ; KARARA, 1971), já implementado no DVideo ${ }^{\circledR}$. Para a determinação da acurácia do sistema, uma haste de $28,64 \mathrm{~cm}$ com marcadores retro-refletivos esféricos nas extremidades foi movimentada no volume calibrado. Após a medição da posição desses marcadores da reconstrução tridimensional ao longo do tempo, o valor de acurácia encontrado foi de $3,91 \mathrm{~mm}$. Os dados tridimensionais foram suavizados com filtro Loess (CUNHA; LIMA FILHO, 2003). A suavização e o cálculo das variáveis foram feitos em ambiente Matlab ${ }^{\circledR}$.

\section{Variáveis Calculadas}

Foram calculados os valores máximos e mínimos dos ângulos do quadril (ângulo absoluto da coxa em relação ao eixo horizontal), do joelho (ângulo relativo entre a coxa e a perna) e do tornozelo (ângulo relativo entre o segmento perna e o segmento pé) ao longo de cinco ciclos de pedalada, em cada método de ajuste de altura de selim, conforme representado na figura 1 . 


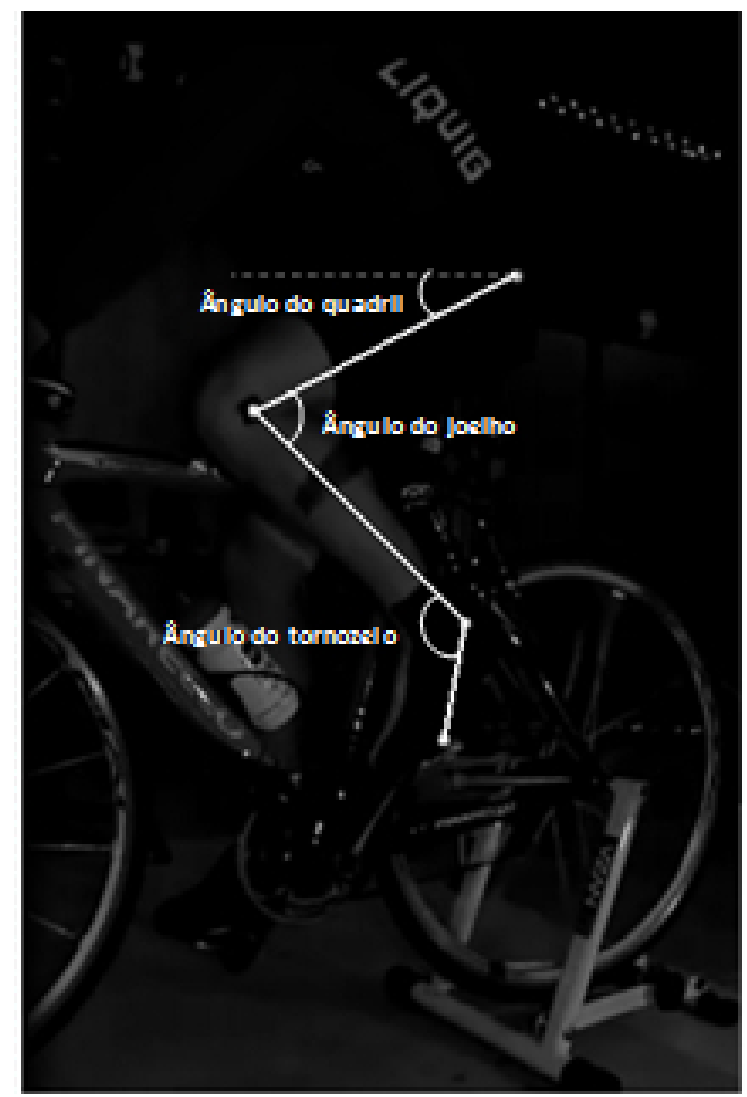

Figura 1: Representação dos ângulos calculados.

Tratamento Estatístico

Os dados relativos aos valores de ajuste de selim encontrados para cada participante serão apresentados de forma descritiva. Para os ângulos máximos e mínimos de quadril, joelho e tornozelo nos ciclos de pedalada em cada tipo de ajuste serão descritos os valores de média e desvio padrão.

\section{Resultados}

$\mathrm{Na}$ tabela 1 estão descritos os valores de altura de selim $(\mathrm{cm})$ obtidos para os ajustes de Greg Lemond e Fit Kit para cada um dos três participantes do experimento. 
Tabela 1: Valores de altura do selim $(\mathrm{cm})$ obtidos pelos métodos de ajuste de Greg Lemond e Fit Kit.

\begin{tabular}{ccc}
\hline Ajuste & Participante & Altura do Selim $(\mathrm{cm})$ \\
\hline \hline \multirow{2}{*}{ Greg } & 1 & 91.35 \\
Lemond $*$ & 2 & 89.05 \\
& 3 & 86.05 \\
Fit Kit & 1 & 89.7 \\
& 2 & 85.8 \\
& 3 & 83.2 \\
\hline
\end{tabular}

*Aos valores obtidos no método Greg Lemond foi somado um fator de correção de $17,25 \mathrm{~cm}$

A tabela 2 apresenta a média ( \pm desvio padrão) dos valores máximos e mínimos encontrados para os ângulos do quadril, joelho e tornozelo nos 5 ciclos de pedalada, analisados nos ajustes de selim Greg Lemond e Fit Kit. Os resultados obtidos para os ângulos não apresentaram grandes diferenças entre os dois tipos de ajuste.

Tabela 2: Valores médios (+/- desvio padrão) dos ângulos máximos e mínimos do quadril, joelho e tornozelo obtidos para os 5 ciclos de pedalada nos ajustes de selim pelos métodos Greg Lemond e Fit Kit.

\begin{tabular}{|c|c|c|c|c|c|c|c|}
\hline \multirow[b]{2}{*}{ Ajuste } & \multirow[b]{2}{*}{ Participante } & \multicolumn{2}{|c|}{$\begin{array}{l}\text { Ângulo do } \\
\text { Quadril }\left({ }^{\circ}\right)\end{array}$} & \multicolumn{2}{|c|}{$\begin{array}{c}\text { Ângulo do Joelho } \\
\left({ }^{\circ}\right)\end{array}$} & \multicolumn{2}{|c|}{$\begin{array}{c}\text { Ângulo do Tornozelo } \\
\left({ }^{\circ}\right)\end{array}$} \\
\hline & & Max. & Min. & Max. & Min. & Max. & Min. \\
\hline \multirow{4}{*}{$\begin{array}{l}\text { Greg } \\
\text { Lemond }\end{array}$} & 1 & $\begin{array}{c}66,1 \\
( \pm 0,8)\end{array}$ & $\begin{array}{c}23,5 \\
( \pm 0,3)\end{array}$ & $\begin{array}{l}142,1 \\
( \pm 1,5)\end{array}$ & $\begin{array}{c}67,1 \\
( \pm 0,7)\end{array}$ & $\begin{array}{l}119,4 \\
( \pm 0,7)\end{array}$ & $\begin{array}{l}101,3 \\
( \pm 1,3)\end{array}$ \\
\hline & 2 & $\begin{array}{c}60,3 \\
( \pm 0,3)\end{array}$ & $\begin{array}{c}21,8 \\
( \pm 0,3)\end{array}$ & $\begin{array}{r}141,0 \\
(+0,4)\end{array}$ & $\begin{array}{r}69,3 \\
(+0,0\end{array}$ & $\begin{array}{l}124,6 \\
(+0,9)\end{array}$ & $\begin{array}{r}98,0 \\
(+077\end{array}$ \\
\hline & & 60,2 & 20,6 & 137,3 & 70,9 & 114,0 & 105,5 \\
\hline & 3 & $( \pm 0,5)$ & $( \pm 0,4)$ & $( \pm 0,7)$ & $( \pm 0,6)$ & $( \pm 0,3)$ & $( \pm 1,0)$ \\
\hline \multirow{3}{*}{ Fit Kit } & 1 & $\begin{array}{c}66,0 \\
( \pm 0,7)\end{array}$ & $\begin{array}{c}23,3 \\
( \pm 0,2)\end{array}$ & $\begin{array}{l}143,9 \\
( \pm 0,9)\end{array}$ & $\begin{array}{c}68,8 \\
( \pm 0,4)\end{array}$ & $\begin{array}{r}121,2 \\
( \pm 0,8)\end{array}$ & $\begin{array}{l}103,6 \\
( \pm 0,6)\end{array}$ \\
\hline & 2 & $\begin{array}{l}66,0 \\
(0,7)\end{array}$ & $\begin{array}{c}23,3 \\
( \pm 0,2)\end{array}$ & $\begin{array}{l}134,2 \\
( \pm 0,2)\end{array}$ & $\begin{array}{c}66,2 \\
( \pm 0,3)\end{array}$ & $\begin{array}{l}125,7 \\
( \pm 4,1)\end{array}$ & $\begin{array}{l}100,2 \\
( \pm 1,3)\end{array}$ \\
\hline & 3 & $\begin{array}{c}60,3 \\
( \pm 0,6)\end{array}$ & $\begin{array}{c}20,4 \\
( \pm 0,4)\end{array}$ & $\begin{array}{l}137,8 \\
( \pm 0,5)\end{array}$ & $\begin{array}{c}70,2 \\
( \pm 0,2)\end{array}$ & $\begin{array}{l}114,1 \\
( \pm 1,5)\end{array}$ & $\begin{array}{l}103,9 \\
( \pm 2,1)\end{array}$ \\
\hline
\end{tabular}

\section{Discussão}

A análise de um movimento esportivo, seja ela de forma bidimensional ou tridimensional, na tentativa de quantificação de variáveis cinemáticas, tem como importante objetivo um melhor entendimento 
sobre as características relacionadas com o desempenho tanto de atletas de alto nível quanto praticantes comuns.

No ciclismo, o ajuste adequado do selim da bicicleta é considerado parte importante da prática, uma vez que interfere no desempenho e na prevenção de lesões (BURKE; PRUITT, 1994; PEVELER et al., 2005). Desta forma, a ergometria durante a pedalada é empregada no sentido de possibilitar a prática de um exercício aeróbio sob condições controladas de carga de trabalho (MCLEOD; BLACKBURN, 1980). A altura do selim vem sendo descrita como um dos mais importantes componentes de configuração da bicicleta que afeta a cinemática e dinâmica dos membros inferiores (DE VEY MESTDAGH, 1998; TAMBORINDEGUY; RICO BINI, 2011).

Além da altura, o ângulo do tubo de assento é outro ajuste que tem sido abordado em estudos da literatura e representa o quanto o selim está deslocado para frente ou para trás. No entanto, um estudo recente mostrou que mudanças no ângulo do tubo de assento possuem pouca influência na potência da pedalada (RANKIN; NEPTUNE, 2010). Desta forma, no presente estudo optou-se pelo ajuste da altura do selim, por ser uma configuração constantemente realizada por ciclistas e de fácil aplicação.

Para esta pesquisa, foram recrutados três sujeitos com pequenas diferenças nas características antropométricas, apesar da grande diferença entre as idades. No entanto, uma vez que a proposta do estudo era avaliar a influência de diferentes ajustes de altura de selim em diferentes indivíduos, a distinção de idade, massa e altura dos participantes não se torna relevante.

Dentre os diversos métodos disponíveis na literatura para ajuste de altura de selim, dois foram comparados no presente estudo. O primeiro tratou-se do método Greg Lemond (BURKE; PRUITT, 2003, BURKE, 1994; PEVELER et al., 2005; PEVELER et al., 2007), de fácil aplicação, cujo ajuste depende apenas da medida de altura entre o chão e o púbis. Por este motivo, trata-se de um método passível de ser utilizado tanto por profissionais quanto praticantes recreacionais. Por outro lado, o Fit Kit é um ajuste comercial, subordinado a um maior número de parâmetros para sua configuração e, por depender de profissionais qualificados para sua aplicação, torna-se um método de custo oneroso, que justifica o baixo número de participantes envolvidos neste estudo. 
Mesmo que cada ajuste leve em consideração variáveis específicas, como uma constante para o Greg Lemond e uma medida de flexibilidade para o Fit Kit, as alturas de selim encontradas para cada participante foram próximas (diferença de 2,2 $\mathrm{cm}$ em média). Este resultado torna-se relevante uma vez que pequenas mudanças na altura do selim não resultam em efeitos significantes sobre cargas na articulação do joelho (TAMBORINDEGUY; RICO BINI, 2011).

Peveler et al. (2005) também não encontraram diferenças entre as alturas sugeridas pelo método Greg Lemond e outros averiguados pela literatura, como o Hamley e calcanhar-pedal. A partir dos resultados mostrados no presente experimento, um método simples e de fácil cálculo como o Greg Lemond pode beneficiar todas as categorias de praticantes de ciclismo, que tem a possibilidade de encontrar qual é o ajuste mais adequado de forma simples e barata.

Os valores mínimos e máximos encontrados para as articulações do quadril, joelho e tornozelo também não apresentaram grandes mudanças com a variação dos diferentes ajustes de altura de selim. Dos três participantes envolvidos na pesquisa, o participante 2 foi o que apresentou maiores mudanças cinemáticas, com destaque para uma diminuição de 6,8 graus na média dos valores máximos do joelho. No entanto, de acordo com um estudo recente (TAMBORINDEGUY; RICO BINI, 2011), esta mudança também não é suficiente para provocar mudanças na força produzida nas articulações.

Os valores de ângulos encontrados podem variar quando comparados com outros trabalhos apresentados na literatura. Os ângulos mínimos e máximos do quadril encontrados nesta pesquisa se assemelham

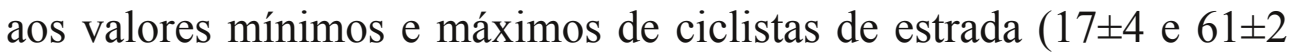
graus, respectivamente) e de ciclistas de mountain-bike ( $23 \pm 3$ e $61 \pm 3$ graus, respectivamente) encontrados na literatura (CARPES et al., 2006). Umberger, Scheuchenzuber e Manos (1998) também apresentaram valores de ângulos do quadril em durante a pedalada, no entanto, no referido estudo, os autores optaram pela representação do ângulo relativo entre a coxa e o tronco, o que inviabiliza a comparação entre os resultados do presente estudo. O ângulo relativo entre a coxa e o tronco sofre forte influência de outros ajustes da bicicleta como, por exemplo, a posição do guidão da bicicleta. Em outras palavras, posicionar o guidão mais próximo do ciclista ou mais afastado provoca mudanças na inclinação do tronco e, consequentemente, produz alterações no ângulo relativo entre a coxa e o tronco. Como a proposta 
deste estudo era analisar a influência de diferentes ajustes na cinemática dos membros inferiores, optou-se pelo ângulo absoluto do quadril e não o relativo.

Por outro lado, os valores máximos do ângulo do joelho encontrados para o ajuste Greg Lemond foram mais baixos com relação ao que já foi reportado por na literatura (PEVELER et al., 2005). Essas diferenças podem se justificar pelas diferentes metodologias utilizadas. Em Peveler et al. (2005), os ângulos articulares foram aferidos por meio de um goniômetro, com o pedal na posição de ponto morto inferior, enquanto o presente utilizou um método de rastreamento automático de marcadores a partir de imagens do movimento de interesse. Este tipo de ferramenta tem como uma de suas vantagens a alta precisão nas medidas (BARRIS; BUTTON, 2008), como pode ser verificado pelo valor de acurácia obtido no presente trabalho $(3,91 \mathrm{~mm})$. Os resultados obtidos corroboram com os encontrados por Carpes et al. (2006), que também usou da videogrametria para a obtenção da posição dos marcadores em função dos ciclos de pedalada.

Estudos sugerem que o ângulo máximo do joelho deve variar entre $145^{\circ}$ e $155^{\circ}$ graus, uma vez que essa faixa de valores apresenta-se como a mais segura na prevenção de lesões, na economia de energia e, consequentemente, melhora do desempenho (BURKE; PRUITT, 1994; PEVELER et al., 2005). Os ajustes realizados neste trabalho refletiram em ângulos de joelho abaixo dos limites estabelecidos pela literatura para essa faixa de segurança. Por outro lado, um estudo atual (RANKIN; NEPTUNE, 2010) aponta que valores ótimos de ângulo de joelho, para a produção de maior potência durante a pedalada, devem ser de $59,6^{\circ}$ e $153,6^{\circ}$ (valor mínimo e máximo, respectivamente). Portanto, ressalta-se que, para estes participantes, ambos os métodos não foram eficazes em provocar valores ótimos de ângulo do joelho para prevenção de lesão ou para melhoria de rendimento, conforme os valores sugeridos pela literatura. Estes resultados servem de alerta para profissionais da área de ergonomia, que atuam em ajustes de bicicletas para ciclistas profissionais e recreacionais, embora uma amostra maior seja necessária para a confirmação dos resultados encontrados.

Os valores de ângulos mínimos e máximos encontrados no presente estudo não corroboram aos apresentados Carpes et al. (2006), que apresentaram valores inferiores para esta articulação. No entanto, estes autores analisaram a pedalada de profissionais do ciclismo de es- 
trada e mountain-bike que possuíam seu próprio ajuste de altura de selim, sendo esta a provável razão da diferença entre os resultados.

Embora os diferentes ajustes não tenham apresentado grandes mudanças na cinemática de membros inferiores durante a pedalada, outros fatores não considerados neste estudo podem ter grande influência no desempenho do ciclista. Dentre eles, destaca-se a inclinação do tronco para uma posição mais aerodinâmica para diminuir a área frontal do ciclista (RANKIN; NEPTUNE, 2010). Nesse sentido, estudos envolvendo uma quantidade maior de participantes, com análises específicas de outros parâmetros de ajustes de bicicleta podem melhor esclarecer estes fenômenos e dar melhor suporte a profissionais da área, pesquisadores e praticantes de ciclismo.

\title{
Conclusão
}

O presente trabalho teve como objetivo esclarecer a influência de dois diferentes métodos de ajuste de altura de selim em variáveis cinemáticas angulares de membro inferior na pedalada. Os resultados demonstraram que a altura do selim definida pelo método Fit Kit não mostrou ângulos de quadril, joelho e tornozelo diferentes daqueles observados quando a altura foi ajustada pelo método Greg Lemond, durante alguns ciclos de pedalada. Desta forma, concluímos que, uma vez que o Fit Kit é um método de alto custo, praticantes de ciclismo podem realizar ajustes na altura do selim da bicicleta através de um método de cálculo simples e de fácil execução, como o Greg Lemond.

The angle variation of the hip, knee and ankle between two methods of adjusting height of bike saddle: case studies

\begin{abstract}
The aim of the present study was evaluate the Greg Lemond method and Fit Kit in lower limbs angular kinematic variables during cycling. It was recruited 3 trained cyclists, who cycled by 2 minutes, in preferred cadence. The images were obtained by 2 digital cameras. Markers were fixed at greater trochanter, lateral epicondyle, lateral malleolus and the base of 5th metatarsal, than tracked in DVideo ${ }^{\circledR}$. We calculated maximum and minimum values of hip, knee and ankle angles during $5 \mathrm{cy}-$ cles. The results showed no difference between the tested methods. Thus, once Fit Kit is an onerous method, it is possible to adjust saddle height using a simple method, as Greg Lemond.
\end{abstract}

Keywords: Cycling. Lower extremity. Biomechanics.

Pensar a Prática, Goiânia, v. 16, n. 1, p. 1-319, jan./mar. 2013 
Las variaciones angulares del cuadril, rodilla y tobillo entre dos métodos de ajuste de altura del sillín de la bicicleta: estudios de caso

\section{Resumen}

El objetivo del estudio fue evaluar las consecuencias de los métodos Greg Lemond y Fit Kit en variables cinemáticas angulares de la extremidad inferior. Fueron seleccionados 3 ciclistas entrenados para pedalear durante 2 minutos en su cadencia preferida. Las imágenes fueron obtenidas por dos videocámaras en una frecuencia de $300 \mathrm{~Hz}$. Fueron fijados marcadores en el trocánter mayor, epicóndilo lateral, maléolo lateral y base del $5^{\circ}$ metatarso que fueron rastreados en el DVideo ${ }^{\circledR}$. Se calcularon los valores máximos y mínimos de los ángulos del cuadril, rodilla y tobillo en 5 ciclos. Los resultados no difieren de los métodos testados. Así, ya que el Fit Kit es un método costoso, es posible la realización de ajustes utilizando un método simple, como el Greg Lemond.

Palabras clave: Ciclismo. Extremidad inferior. Biomecánica.

\section{Referências}

ABDEL-AZIZ, Y. I.; KARARA, H. M. Direct linear transformation from comparator coordinates into object-space coordinates. In: ASP/UI SYMPOSIUM ON CLOSE-RANGE PHOTOGRAMMETRY, 1971, Illinois. Proceedings. Falls Church, VA: American Society of Photogrammetry, 1971. p.1-18.

ALENCAR, T. A. M.; MATIAS, K. F. S. Importância da avaliação musculoesquelética e biomecânica para o fit bike. Movimenta, Goiás, v. 2, n. 3, p. 84-92, 2009.

ALENCAR, T. A. M.; MATIAS, K. F. S. Bike Fit e sua importância no ciclismo. Movimenta, Goiás, v. 2, n. 2, p. 59-64, 2009.

BAILEY, M. P.; MAILLARDET, F. J.; MESSENGER, N. Kinematics of Cycling in Relation to Anterior Knee Pain and Patellar Tendinitis. Journal of Sports Sciences, v. 21, n. 8 p. 649-57, 2003.

BARRIS, A.; BUTTON, C. A. Review of Vision-Based Motion Analysis in Sport. Sports Medicine, vol. 38, n. 12, p. 1025-1043, dez., 2008.

BARROS, R. M. L. et al. Desenvolvimento e avaliação de um sistema para análise tridimensional de movimentos humanos. Revista Brasileira de Engenharia Biomédica, Campinas, vol. 15, n. 1-2, p. 79-86, 1999. 
BINI, R.; HUME, P. A.; CROFT, J. L. Effects of bicycle saddle height on knee injury risk and cycling performance. Sports Medicine, vol. 41, n. 6, p. 463-476, 2011.

BOUCHÉ, R. T.; VINCENT, P.M.; SULLIVAN, K. Bike fit evaluation: Can it help diagnose and prevent cycling injuries? Podiatry Today, Malvern, v. 19, n. 12, p. 28-34, 2006.

BURKE, E. R. Proper Fit of the Bicycle. Clinical Sports Medicine, v. 13 n.1 p. 1-14, 1994.

BURKE, E. R.; PRUITT, A. L. Body Positioning for Cycling. In: BURKE, E. R. High-Tech Cycling. $2^{\text {a }}$ ed. Champaign, IL: Human Kinetics, 2003. p. 69-92

CARPES, F. et al. Características cinemáticas da pedalada em ciclistas competitivos de diferentes modalidades. Revista Portuguesa de Ciências do Desporto, Portugal, v. 6, n. 1, p. 07-14, 2006.

CUNHA, S. A.; LIMA FILHO, E. C. Metodologia para suavização de dados biomecânicos por função não paramétrica ponderada local robusta. Revista Brasileira de Biomecânica, São Paulo, v.1, n.6, p.2328. 2003.

DE VEY MESTDAGH, K. Personal perspective: in search of an optimum cycling posture. Applied Ergonomics, v.29, n.5, p.325-34, out., 1998.

FIGUEROA, P. J.; LEITE, N.; BARROS, R. M . L. Flexible software for tracking markers for human motion analyses. Computer Methods Programs in Biomedicine, vol.72, n. 2, p. 155-165, out., 2003.

HOLMES, J. C.; PRUITT, A. L.; WHALEN, N. J. Low extremity overuse in bicycling. Clinical Sports Medicine, v. 13 n. 1, p. 187205, 1994.

MARTINS, E. A. et al. Avaliação do posicionamento corporal no ciclismo competitivo e recreacional. Revista Brasileira de Cineantropometria e Desempenho Humano, Florianópolis, v. 9, n. 2, p. 183-188, 2007. 
MCLEOD, W. D. e BLACKBURN, T. A. Biomechanics of knee rehabilitation with cycling. American Journal of Sports Medicine, Chicago, v.8, n.3, maio/jun., p.175-180. 1980.

MESTDAGH, K. V. Personal perspective: in search of an optimum cycling posture. Applied Ergonomics, v. 29, n. 5, p. 325-334, out., 1998.

PEVELER, W. Effects of Saddle Height on Economy in Cycling. Journal of Strength and Conditioning Research, v. 22, n. 4, p.1355-1359, jul., 2008.

PEVELER, W. et al. Comparing methods for setting saddle height in trained cyclists. Journal of Exercise Physiology, v. 8, n. 1, p. 51-55, 2005.

PEVELER, W.; POUNDERS, J. D.; BISHOP, P. A. Effects of Saddle Height on Anaerobic Power Production in Cycling. Journal of Strength and Conditioning Research, v. 21 n. 4 p. 1023-1027, nov., 2007.

RANKIN, J. W. E NEPTUNE, R. R. The influence of seat configuration on maximal average crank power during pedaling: a simulation study. Journal of Applied Biomechanics, v.26, n.4, p.493-500, nov., 2010.

SILBERMAN, M.R et al. Road bicycle fit. Clinical Journal of Sport Medicine, v. 15, n. 4, p. 271-276, jul., 2005.

TAMBORINDEGUY, A. C. E; RICO BINI, R. Does saddle height affect patellofemoral and tibiofemoral forces during bicycling for rehabilitation? Journal of Bodywork and Movement Therapies, v.15, n.2, p.186191, abr., 2011.

UMBERGER, B. R.; SCHEUCHENZUBER, H. J. E; MANOS, T. M. Differences in power output during cycling at different seat tube angles. Journal of Human Movement Studies, v.35, p.21-36. 1998.

Recebido em: 13/10/2011

Revisado em: 05/03/2012

Aprovado em: 24/07/2012

Pensar a Prática, Goiânia, v. 16, n. 1, p. 1-319, jan./mar. 2013 


\section{Endereço para correspondência}

sacunha@fef.unicamp.br

Sergio Augusto Cunha

Universidade Estadual de Campinas

Faculdade de Educação Física, Departamento de Ciências do Esporte.

Av. Érico Veríssimo, 701- Cidade Universitária Zeferino Vaz

Cidade Universitáira

13083-851 - Campinas, SP - Brasil - Caixa-postal: 6134 